\title{
Liderazgo pedagógico para reestructurar creencias docentes y mejorar prácticas de aula en contexto mapuche
}

\author{
Pedagogical Leadership to Restructure Teaching Beliefs and Improve Classroom \\ Practices in Mapuche Context
}

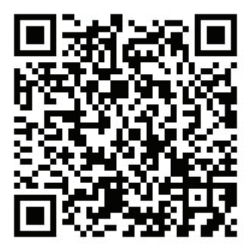

\author{
María Elena Mellado-Hernández ${ }^{1}$ \\ Universidad Católica de Temuco \\ Temuco, Chile \\ mmellado@uct.cl \\ Juan Carlos Chaucono-Catrinao \\ Escuela Primaria de Vilcún \\ Temuco, Chile \\ juancarchau@yahoo.es
}

Recibido 10 de enero de 2015 • Corregido 7 de noviembre de 2015 • Aceptado 3 de noviembre de 2015

\footnotetext{
${ }^{1}$ Doctora en Formación de Profesores de la Universidad de Extremadura, España. Magíster en Informática Educativa de la Universidad de la Frontera. Profesora de Educación General Básica de la Pontificia Universidad Católica de Chile. Dedicada en las últimas dos décadas a formar docentes tanto en pregrado como en postgrado en la Universidad Católica de Temuco, Chile. Además, se desempeña como investigadora en las áreas de liderazgo escolar y formación de profesorado. Sus publicaciones versan sobre temáticas como creencias del profesorado, e-portafolio y rúbrica, reflexión de la práctica pedagógica, evaluación para el aprendizaje y liderazgo pedagógico. Profesora titular de la carrera de Pedagogía de Educación Básica. Actualmente directora del Magíster en Gestión Escolar en el contexto del Plan de Formación de Directores del Ministerio de Educación de Chile.

2 Magister en Gestión Escolar de la Universidad Católica de Temuco, Licenciado en Educación y Profesor de Educación General Básica con especialidad en Ciencias Naturales de la Pontificia Universidad Católica de Chile. Dedicado en la última década a formar estudiantes de educación general básica en contexto rural mapuche e investigar en esta área en el contexto del Magíster en Gestión Escolar de la Universidad Católica de Temuco. Actualmente se desempeña como director de una escuela particular subvencionada de la comuna de Vilcún, región de La Araucanía, Chile.
} 
doi: http://dx.doi.org/10.15359/ree.20-1.18

URL: http://www.una.ac.cr/educare

CORREO: educare@una.cr

Resumen. Este artículo pretende describir las creencias pedagógicas sobre enseñanza, aprendizaje y evaluación que subyacen a las prácticas de enseñanza en una escuela rural mapuche, región de La Araucanía, Chile. El estudio se suscribe en un enfoque de investigación educativa, utiliza una metodología mixta de carácter descriptiva. Los participantes son profesionales en puestos directivos y en docencia de una escuela que se desempeña desde el nivel transición hasta $6^{\circ}$ año básico en las asignaturas de Lenguaje y comunicación e historia, Geografía y ciencias sociales. Para recoger información sobre las creencias se utilizó un cuestionario de escala tipo Likert; además se realizó observación de clases a través de registros etnográficos a todo el personal docente del centro. A partir de los resultados fue posible identificar contradicciones en los marcos de referencia en ambas poblaciones, pues expresan tanto creencias pedagógicas tradicionales como contemporáneas. Mientras, en el aula se observan prácticas de enseñanza solo tradicionales que ignoran el contexto mapuche, presentan actividades de aprendizaje memorísticas y utilizan de preferencia la evaluación sumativa. En consecuencia, se puede concluir que cuando el profesorado presenta ambigüedades de concepciones en sus creencias pedagógicas predomina un enfoque tradicional en sus prácticas de aula. Por tanto, se recomienda desarrollar un liderazgo pedagógico efectivo que propicie sistemáticamente la reflexión colaborativa entre pares como una estrategia que posibilite la reconstrucción de las creencias pedagógicas de directivos, directivas y docentes.

Palabras claves. Concepciones pedagógicas, creencias del profesorado, práctica docente, liderazgo pedagógico, reflexión pedagógica.

Abstract. This article aims to describe the pedagogical beliefs about teaching, learning and assessment underlying the practices of teaching in a rural Mapuche school, located in La Araucania, Chile. The study is executed in an educational research approach, uses a mixed descriptive character methodology. Participants are professionals in managerial and teaching positions in a school that includes transitional level up to 6th grade in the subjects of language/communication and history, geography and social sciences. To collect information on the beliefs, a questionnaire of Likert type scale was used; also, class observations were carried out through ethnographic records to all teachers of the center. Based on the results, it was possible to identify contradictions in the reference frames in both populations because they express both traditional and contemporary pedagogical beliefs. Meanwhile, in the classroom only traditional teaching practices that ignore the Mapuche context are observed. They show rote learning activities and use, preferably, summative evaluation. As a result, it can be concluded that teachers have ambiguities of conceptions in their pedagogical beliefs, it prevails a traditional approach in their classroom practices. Therefore, it is recommended to develop an effective educational leadership that systematically fosters collaborative thinking among peers as a strategy to enable the reconstruction of educational beliefs of principals and teachers.

Keywords. Pedagogical beliefs, teachers'beliefs, teaching practice, educational leadership, educational thinking. 
doi: http://dx.doi.org/10.15359/ree.20-1.18

URL: http://www.una.ac.cr/educare

CORREO: educare@una.cr

En Chile actualmente se considera como principal problemática la calidad de la educación. La reforma en marcha tiene como factores claves, desarrollar las prácticas directivas y del profesorado para mejorar, de manera efectiva, la enseñanza y el aprendizaje que ocurre en las aulas. En este sentido, Leithwood (2009) sostiene la necesidad de un liderazgo centrado en el desarrollo de las personas que favorezca el aprendizaje, tanto del equipo docente como del estudiantado.

En las últimas décadas se han implementado diversos programas de perfeccionamiento impulsados por la política educativa que no han logrado impactar en las formas de enseñar del personal docente. Estas iniciativas de formación, generalmente versan sobre el saber disciplinario y pedagógico. Sin embargo, estos procesos formativos no han sido significativos porque desconocen las creencias que trae el profesorado (Erazo-Jiménez, 2009).

Por otra parte, los resultados durante la última década en la dimensión de reflexión pedagógica que obtiene el profesorado en la evaluación docente que se realiza en Chile, alcanzan un nivel reflexivo anecdótico (Sun, Correa, Zapata y Carrasco, 2011). En tanto, el Gobierno de Chile, Agencia de la calidad de la educación (2015) informa que los niños y niñas logran bajos resultados en la mayoría de las pruebas estandarizadas. En otras palabras, el cuerpo docente requiere desarrollar procesos reflexivos más críticos sobre la práctica pedagógica que impacten efectivamente en los resultados de aprendizaje del estudiantado.

De igual forma, diversas investigaciones progresivamente han señalado que las creencias pedagógicas se han convertido en un filtro para la mejora y el cambio educativo (Díaz, Martínez, Roa y Sanhueza, 2010; Jiménez y García, 2006; Rojas, 2014). En cambio, Brown (2004, citado en Blázquez y Tagle, 2010) señala que se modificarían las creencias a través de un proceso de reflexión sobre estas mismas. Por consiguiente, la reflexión permanente de la práctica pedagógica y el análisis de las creencias que subyacen al desempeño docente juegan un papel preponderante en posibilitar su transformación y mejorar la eficiencia del profesorado.

En este sentido, varios estudios explican que para reconceptualizar las creencias directivas y docentes es preciso asumir una visión conjunta del liderazgo pedagógico que propicie una gestión colaborativa que conduzca hacia una comunidad profesional de aprendizaje (Bolívar-Botía, 2010a; Day y Gu, 2012; Leithtwood y Day, 2007). En esta línea, Bolívar-Botía (2010b) afirma que develar las creencias pedagógicas que subyacen a la práctica de aula contribuye a tomar decisiones orientadas a mejorar el desempeño profesional. En otras palabras, la reestructuración de las concepciones docentes requiere de líderes pedagógicos que transformen el centro educativo en un espacio de reflexión democrático que propicie el cuestionamiento del pensamiento de los grupos profesionales que se desempeñan en las escuelas. 
doi: http://dx.doi.org/10.15359/ree.20-1.18

URL: http://www.una.ac.cr/educare

CORREO: educare@una.cr

En este marco, el presente artículo tiene por objetivo identificar las creencias contradictorias de enseñanza, aprendizaje y evaluación, según enfoque pedagógico, expresadas por personal directivo y docente. Asimismo, se pretenden describir las prácticas pedagógicas que utiliza el profesorado en el aula. Finalmente, se busca relacionar las creencias evaluativas contradictorias con las prácticas de aula del profesorado.

\section{Referentes conceptuales}

Gestionar centros educativos desde una perspectiva burocrática centrada en la administración sigue siendo hoy una realidad en muchas de las escuelas chilenas. Sin embargo, en la última década ha surgido un reconocimiento al impacto que provoca el liderazgo escolar en las prácticas pedagógicas y en el aprendizaje estudiantil. Por ello, el liderazgo pedagógico se ha convertido en un nudo crítico en la mejora escolar (Bolívar-Botía, 2010a).

Por consiguiente, existen diversas investigaciones que sostienen que un directivo con capacidades de liderazgo pedagógico transforma la cultura de la escuela y hace partícipe al equipo docente de un rol protagónico en la mejora escolar con foco en el aprendizaje del estudiantado (Aaronson, Barrow y Sander, 2007; Barber y Mourshed, 2008; Murillo, 2006; Rivkin, Hanushek y Kain, 2005). En tal sentido, Barber y Mourshed (2008), Leithwood, Harris y Hopkins (2008) y Mulford (2006) sitúan al liderazgo educativo como el segundo factor interno en la escuela que más relevancia tiene en los logros de los aprendizajes, tras el quehacer pedagógico docente. Por consiguiente, resulta imperativo desarrollar capacidades de liderazgo escolar que impulsen prácticas de gestión centradas en el aprendizaje.

En este sentido, varios autores afirman que las creencias pedagógicas se han convertido en objeto de investigación clave para la comprensión de las prácticas directivas y docentes (Antúnez y Aranguren, 2004; Clark, 2008; Gil, Rico y Fernández, 2002; Gómez y Seda, 2008; Mellado y Chaucono, 2015). Según Contreras (2010) las creencias son elementos fundamentales que estructuran el conocimiento profesional docente. En otras palabras, las creencias coexisten en el pensamiento y forman parte de la estructura cognitiva del personal docente, se despliegan en las acciones y decisiones que giran en torno al aula.

La reestructuración de las creencias pedagógicas se pueden modificar si estas fueron construidas a nivel discursivo o si se presentan ambiguamente en los marcos de referencia del profesorado (Gómez, et al., 2014). En este sentido, Moreno (2000) y Prieto (2008) plantean que es necesario explicitar las creencias y reflexionar en torno a ellas para superar las barreras que obstaculizan el proceso de aprendizaje del estudiantado. En este contexto, la reflexión sistemática del profesorado juega un factor clave en develar el pensamiento pedagógico y desde ese conocimiento mejorar el desempeño docente en el aula. 
doi: http://dx.doi.org/10.15359/ree.20-1.18

URL: http://www.una.ac.cr/educare

CORREO: educare@una.cr

Distintos estudios han evidenciado el carácter subjetivo de las creencias docentes, construidas en contexto social, y que tienden a permearse por fuertes componentes afectivos y valorativos (Contreras, 2010; Llinares, 1991; Rojas, 2014). Por otro lado, Martínez, Montero y Pedrosa (2009) afirman que las creencias se yuxtaponen con las dudas, conocimientos y adquisición, las cuales se entrelazan con distintos niveles de complejidad. En otras palabras, las creencias pedagógicas del cuerpo docente no son un continuo lineal, ya que pueden presentarse en el pensamiento del educador o educadora a través de un sincretismo de conocimientos disciplinarios y pedagógicos fragmentados.

Las recientes investigaciones explican que las creencias responden a una lógica y perspectiva que suele mimetizarse a un referente epistemológico que se expresa en concepciones o saberes híbridos que confluyen en el pensamiento del profesorado (García, 2010; Gómez, 2010; Mellado y Chaucono, 2015). En este sentido, Prieto (2008) reconoce que explicitar las creencias pedagógicas y reflexionar sobre ellas mejora la calidad de la práctica docente. Es decir, las representaciones implícitas pueden manifestarse a través de una constante reflexión que muestre las contradicciones pedagógicas que poseen los cuerpos directivos y docentes siempre que se tensionen con el conocimiento teórico y empírico que aporta la bibliografa.

Diversos autores han consensuado el enfoque pedagógico como una forma de entender y actuar en la construcción de procesos de aprendizaje para el cambio. Estos enfoques caracterizan las creencias sobre la enseñanza-aprendizaje que subyacen a la práctica del profesorado (Costamagna, 2015; García, 2010). Asimismo, Vera y Meneses (2012) han planteado la adscripción del profesorado a perspectivas constructivistas en coexistencia con creencias de enfoque conductista. En otras palabras, las creencias docentes sobre el proceso de enseñanza aprendizaje aluden a sistemas tradicionales y contemporáneos.

\section{Metodología}

El estudio se suscribe en el enfoque de investigación educativa que utiliza una metodología mixta, de carácter descriptivo, cuyo propósito es describir la práctica educativa que incluye tanto comportamientos observables como los significados e interpretaciones que dicha práctica lleva asociados para quienes la realizan (Amaya, 2007).

\section{Participantes}

La investigación se realizó en una escuela primaria en contexto rural mapuche, situada en la comuna de Vilcún de la región de La Araucanía. Participan en este estudio, el equipo directivo y docente que se desempeñan en el centro educativo. Los criterios de selección corresponden a accesibilidad al centro, disposición de participantes para ser observados durante un tiempo prolongado y conocimiento de la cultura mapuche. Los sujetos participantes se distribuyen en dos directivos y cuatro docentes que atienden aulas multigrado que se organizan en transición 
doi: http://dx.doi.org/10.15359/ree.20-1.18

URL: http://www.una.ac.cr/educare

CORREO: educare@una.cr

I y II; primer y segundo año básico; tercer y cuarto año; quinto y sexto básico. Sus edades fluctúan entre 33 y 61 años de edad. Respecto al sexo del equipo directivo corresponde a una persona mujer y la otra es hombre. En relación con el cuerpo docente participan un hombre y tres mujeres con una carga horaria entre 30 a 40 horas. Poseen título de profesor de Educación General Básica con especialización disciplinaria. En cuanto a los años de experiencia hay dos que tienen entre 5 y 10 años y dos con más de 20 años de docencia. Cabe destacar que el 50 $\%$ de participantes del estudio son de ascendencia mapuche y el otro $50 \%$ conoce la cultura, porque se han desempañado por varios años en la zona rural mapuche.

\section{Técnicas de recogida de información}

En este estudio se utilizan las técnicas de recolección de información de encuesta y observación.

En primer lugar, se aplica el cuestionario de escala de valoración tipo Likert, construido y validado por Blázquez y Tagle (2010), posee un nivel de fiabilidad de .81. Este instrumento se estructura en pares de ítems que se presentan en el cuestionario aleatoriamente asociados a enfoques pedagógicos tradicionales y contemporáneos sobre creencias pedagógicas que corresponden a las dimensiones de enseñanza, aprendizaje y evaluación.

En segundo lugar, se utilizó la observación no participante (Anguera, Blanco-Villaseñor y Losada, 2001). La observación se focalizó en tres dimensiones: enseñanza, aprendizaje y evaluación. Se realizaron observaciones directas en el aula, cuatro sesiones de clases de 90 minutos a cada docente durante un semestre académico, tanto en las asignaturas de Lenguaje y Comunicación como en Historia, Geografía y Ciencia Sociales, en total 8 clases alcanzando a 720 horas de registro por cada participante. El procedimiento que se utilizó para recoger la información fueron los registros etnográficos y de audio de las clases para luego poder ampliar los registros y realizar análisis de contenido.

\section{Procedimiento de análisis}

La información recogida, tanto por el cuestionario como del registro de clases, se sometió a un riguroso análisis. En primer lugar, se ordenaron los datos por pares de ítems en una planilla Excel considerando el enfoque pedagógico. Luego para cada dimensión de enseñanza, aprendizaje y evaluación se extraen ejemplos de pares ítems que resultan recurrentes y contradictorios, tanto para personal directivo como docentes, desde los enfoques pedagógicos contemporáneos y tradicionales. En segundo lugar, se procede a realizar un análisis de contenido del registro de clase, en busca de categorías que surgen de la concentración de significados recurrentes en las prácticas de aula observadas. Finalmente, se relacionan algunas creencias evaluativas contradictorias asumidas por directivos y docentes con las actividades observadas en el aula. 


\section{Resultados}

En primer lugar, se presentan las creencias contradictorias de enseñanza, aprendizaje y evaluación, según enfoque pedagógico, compartidas por cuerpos directivos y docentes. Luego, las prácticas pedagógicas que utiliza el profesorado en el aula. Finalmente, se relacionan las creencias evaluativas contradictorias que manifiestan personal directivo y profesorado con las prácticas pedagógicas del aula.

\section{Creencias pedagógicas contradictorias de personal directivo y docente}

En general, directivos y docentes que participan de este estudio evidencian creencias de enseñanza, aprendizaje y evaluación que denotan una contradicción desde un enfoque pedagógico contemporáneo y tradicional.

En relación con la dimensión de creencias sobre enseñanza, expresan contradicciones en los marcos de referencia, tal como muestra la tabla 1.

Tabla 1

Creencias de enseñanza contradictorias expresadas por personal directivo y docente según enfoque pedagógico

\begin{tabular}{ll}
\hline Creencias asociadas a un enfoque contemporáneo & Creencias asociadas a un enfoque tradicional \\
\hline $\begin{array}{l}\text { La enseñanza se diseña a partir del contexto de } \\
\text { estudiantes. }\end{array}$ & $\begin{array}{l}\text { La enseñanza se diseña solo a partir del currículo } \\
\text { prescrito. }\end{array}$ \\
$\begin{array}{l}\text { Las actividades de enseñanza deben adecuarse a las } \\
\text { características de estudiantes. }\end{array}$ & $\begin{array}{l}\text { Las actividades de enseñanza deben ser iguales } \\
\text { para todas las personas. }\end{array}$ \\
$\begin{array}{l}\text { Enseñar consiste en generar las condiciones apropiadas } \\
\text { para que el estudiantado logre el aprendizaje. }\end{array}$ & $\begin{array}{l}\text { Enseñar consiste en trasmitir información a } \\
\text { estudiantes. }\end{array}$ \\
\hline
\end{tabular}

Nota: Elaboración propia.

La tabla 1 muestra las principales contradicciones de enfoques pedagógicos que expresan tanto profesorado como personal directivo. Esta situación refleja que los marcos de referencias son compartidos al pensar que 'la enseñanza se diseña a partir del contexto de los estudiantes', por tanto, se entiende que las personas participantes asumen la construcción del diseño de enseñanza considerando el entorno sociocultural estudiantil. A su vez, declaran que 'la enseñanza se diseña solo a partir del currículo prescrito', la opción por esta afirmación deja en evidencia que entienden el diseño de la enseñanza solo desde el currículo establecido por el Ministerio de Educación obviando el contexto mapuche de sus estudiantes. Del mismo modo, manifiestan 
doi: http://dx.doi.org/10.15359/ree.20-1.18

URL: http://www.una.ac.cr/educare

CORREO: educare@una.cr

que 'las actividades de enseñanza deben adecuarse a las características de los estudiantes', dando a entender que las clases se adecuan a las necesidades, intereses y experiencias previas del estudiantado; sin embargo, también expresan que 'las actividades de enseñanza deben ser iguales para todos' evidenciando claras contradicciones. También, el grupo en estudio declara que 'enseñar consiste en generar las condiciones apropiadas para que los estudiantes logren el aprendizaje, por consiguiente, se podría entender que asumen la clase como una oportunidad de aprendizaje. De igual manera, piensan que 'enseñar consiste en trasmitir información a los estudiantes', restringiendo el aprendizaje a la mera repetición y memorización de contenidos.

En la dimensión de creencias sobre aprendizaje, ambos grupos manifiestan contradicciones en sus marcos de referencia. En tabla 2, se presentan algunos ejemplos que evidencian dichas ambigüedades.

Tabla 2

Creencias de aprendizaje contradictorias expresadas por personal directivo y docente según enfoque pedagógico

\begin{tabular}{ll}
\hline Creencias asociadas a un enfoque contemporáneo & Creencias asociadas a un enfoque tradicional \\
\hline $\begin{array}{l}\text { Las actividades que aparecen en los libros de textos } \\
\text { pueden ser modificadas. }\end{array}$ & $\begin{array}{l}\text { Las actividades que aparecen en los libros de textos } \\
\text { no pueden ser modificadas. }\end{array}$ \\
$\begin{array}{ll}\text { El estudiante aprende cuando construye significados } \\
\text { aplicándolos a nuevas situaciones. }\end{array}$ & $\begin{array}{l}\text { El estudiante aprende cuando reproduce } \\
\text { información. }\end{array}$ \\
\hline
\end{tabular}

Nota: Elaboración propia.

En la tabla 2, se hace referencia a los resultados contradictorios en la dimensión de aprendizaje, al asumir que 'las actividades que aparecen en los libros de textos pueden ser modificadas', pero a su vez, expresan que 'las actividades que aparecen en los libros no pueden ser modificadas'. Estas contradicciones de enfoques dan indicios de que tanto directivos como docentes no poseen claridad si pueden o no adaptar las actividades que aparecen en el texto de estudio al contexto sociocultural de sus estudiantes. Además, manifiestan estar muy de acuerdo con la afirmación 'el estudiante aprende cuando construye significados aplicándolos a nuevas situaciones' y al mismo tiempo, declaran que 'el estudiante aprende cuando reproduce información'. Esta situación discordante, deja entrever que tienen confusiones en sus creencias pedagógicas sobre el aprendizaje.

Asimismo, al analizar los resultados de la dimensión sobre creencias de evaluación, expresados por personal directivo y docente se observan ambigüedades. La tabla 3 presenta algunos ejemplos de estas contradicciones. 
doi: http://dx.doi.org/10.15359/ree.20-1.18

URL: http://www.una.ac.cr/educare

CORREO: educare@una.cr

Tabla 3

Creencias de evaluación contradictorias expresadas por personal directivo y docente según enfoque pedagógico

\begin{tabular}{ll}
\hline \multicolumn{1}{c}{ Creencias asociadas a un enfoque contemporáneo } & Creencias asociadas a un enfoque tradicional \\
\hline $\begin{array}{l}\text { La prueba escrita puede ser utilizada para evaluar } \\
\text { solo ciertas habilidades. }\end{array}$ & $\begin{array}{l}\text { La prueba escrita puede ser utilizada para evaluar } \\
\text { cualquier tipo de habilidad. }\end{array}$ \\
$\begin{array}{ll}\text { La evaluación debe centrarse en valorar la forma en } \\
\text { Lae el estudiantado construye conocimientos en }\end{array}$ & $\begin{array}{l}\text { de información que el estudiantado es capaz de } \\
\text { reproducir. }\end{array}$ \\
diversos contextos. & El estudiantado no debe participar en la construcción \\
El estudiantado puede participar en la construcción & de los criterios de evaluación. \\
de los criterios de evaluación. &
\end{tabular}

Nota: Elaboración propia.

A partir de los resultados presentados en la tabla 3, sobre las creencias de evaluación del aprendizaje expresadas tanto por personal directivo como docente, se evidencian incoherencias en sus marcos de referencia desde el punto de vista del enfoque pedagógico. Por un lado, manifiestan que 'la prueba escrita puede ser utilizada para evaluar solo ciertas habilidades', a su vez, señalan que 'la prueba escrita puede ser utilizada para evaluar cualquier tipo de habilidad', esto refleja que existe una sobrevaloración de la prueba como instrumento objetivo de evaluación. Al mismo tiempo, declaran que'la evaluación debe centrarse en valorar la forma en que los estudiantes construyen conocimientos en diversos contextos' cuando afirman estar de acuerdo con este planteamiento da la impresión que los participantes del estudio utilizan en su práctica evaluativa diversos instrumentos atingentes a las necesidades de estudiantes y que valoran la forma que aprenden. Sin embargo, la contradicción se produce cuando a la vez afirman que'la evaluación debe centrarse en conocer la cantidad de información que el estudiante es capaz de reproducir', esto corrobora que el quehacer evaluativo en los procesos de enseñanza aprendizaje es asociado a la recuperación de información de parte del estudiantado. Del mismo modo, directivos y docentes afirman que 'los estudiantes pueden participar en la construcción de los criterios de evaluación', dando a entender que hacen partícipes de su proceso evaluativo a sus estudiantes. Por consiguiente, manifiestan que 'los estudiantes no deben participar en la construcción de los criterios de evaluación', dando claros indicios que coexisten pensamientos ambiguos sobre el protagonismo del estudiante en el proceso evaluativo. En otras palabras, los resultados permiten inferir que personal directivo y docente de este centro escolar presentan confusiones sobre la concepción evaluativa y parece predominar un enfoque tradicional. 
doi: http://dx.doi.org/10.15359/ree.20-1.18

URL: http://www.una.ac.cr/educare

CORREO: educare@una.cr

En síntesis, estas contradicciones de enfoques pedagógicos en enseñanza, aprendizaje y evaluación dan cuenta que tanto directivos como docentes tienen un pensamiento similar y confuso sobre el saber pedagógico. Por un lado, reconocen algunas afirmaciones más contemporáneas, desde una perspectiva constructivista, pero a su vez, este discurso es anulado por creencias que responden a un enfoque tradicional, más asociado a una postura conductista, que deja ver las incoherencias de conocimiento pedagógico de los sujetos participantes. Es necesario comprender que estos discursos divergentes son asumidos inconscientemente por estos grupos, derivados de su experiencia escolar y de concepciones teóricas tradicionales de enseñar, aprender y evaluar. Es imprescindible que personal directivo y docente tomen conciencia de que sus marcos de referencias afectan las prácticas de liderazgo pedagógico para gestionar la docencia, a nivel de centro y de aula. Por tanto, se requiere de líderes pedagógicos que generen oportunidades de aprendizaje entre profesionales de la comunidad educativa para promover sistemáticamente discusión y cuestionamiento de sus creencias, apoyados por aportes teóricos y empíricos con el propósito de movilizar su pensamiento pedagógico.

\section{Prácticas pedagógicas que utiliza el profesorado en el aula}

El análisis del contenido de los registros de clases permite agrupar la información en tres categorías, que surgen de la concentración de significados comunes y recurrentes en las prácticas observadas. Estas categorías son: contexto de enseñanza, aprendizaje repetitivo y memorístico, y evaluación sumativa. El sistema utilizado para identificar los fragmentos fue: $\mathrm{R}$ para referirnos a registros y $\mathrm{P}$ para nombrar a docentes.

\section{Contexto de enseñanza}

Las actividades de enseñanza observadas generalmente se desarrollan considerando el currículo prescrito, obviando el contexto de enseñanza de ruralidad y de la cultura mapuche. A continuación se presenta un fragmento de clase que evidencia esta situación.

Pa: "Estimados estudiantes, hoy vamos a leer una leyenda de la cultura de una isla grande de Chiloé".

Ao2: "¿Dónde queda esa isla Tía?" (Se produce un silencio en la sala, luego la profesora explica que se ubica al sur de Chile en la Región de los Lagos).

Ao8: "iTía! ¡Tía! Yo leí en un libro la leyenda mapuche del Pehuén"

Pa: "iMuy bien! Efectivamente la leyenda que comenta Rayen existe, pero ahora tenemos que leer El Calueche".

Pa: "Ahora todos leyendo en silencio" (la profesora se pasea entre los puestos y monitorea la lectura, luego entrega una guía con preguntas).

[R 1, P 3] 
doi: http://dx.doi.org/10.15359/ree.20-1.18

URL: http://www.una.ac.cr/educare

CORREO: educare@una.cr

En la práctica descrita se observa una actividad de clases que desconoce el contexto sociocultural, omite los intereses y experiencias previas del estudiante, lo que dificulta la construcción de aprendizaje significativo.

\section{Aprendizaje repetitivo y memorístico}

Se propicia un proceso de aprendizaje memorístico, restringido a extraer información literal del texto. El estudiantado se limita a contestar preguntas explícitas, poco desafiantes que solo exigen recuperar información. Tal como se presenta a continuación:

Po: ¡AAtención niños! “Hoy estudiaremos sobre la geografía de Chile” (el profesor lee y los niños y niñas siguen en silencioso la lectura que se encuentra en el libro de historia y geografía otorgado por el MINEDUC).

Po: "Emilio! ¿Cuáles son las ciudades más grandes de Chile?”

Ao7: "Concepción, Santiago y Antofagasta"

Po: "iBien Emilio!"

Aa3: "Temuco también es grande profesor"

Po: "Alguien me pude decir ¿Qué ciudad está más al norte de Chile?"

Ao8: "ilquique Tío! (grita un niño desde su puesto)

Po: "¡Noo! ¿Cuál está más al norte según la lectura?"

Ao1: "Aquí dice Arica profesor"

Po: "Bien, Arica es la ciudad más al norte de Chile"

$[\mathrm{R} 1, \mathrm{P} 4]$

En el ejemplo anterior, el estudiante asume un rol como agente reproductor de la información en las actividades de clases que aparecen en el texto de estudio. En este sentido, se puede inferir que el docente cree que comprender el texto es solo repetir información explícita, no considera la interpretación a partir de las experiencias y conocimientos previos de del alumnado. Esta práctica de aula basada en preguntas literales desarrolla habilidades básicas del pensamiento y restringe aquellas más complejas.

\section{Evaluación sumativa}

La mayoría de las actividades evaluativas observadas en el aula corresponden a evaluaciones sumativas, están centradas en el resultado de aprendizaje al final del periodo académico. En otras palabras, el docente enseña para colocar nota y el niño realiza su tarea por 
doi: http://dx.doi.org/10.15359/ree.20-1.18

URL: http://www.una.ac.cr/educare

CORREO: educare@una.cr

una calificación. Estas prácticas evaluativas son recurrentes en la cultura escolar y desvían al estudiantado del verdadero sentido del aprendizaje.

Pa: "Recuerden niños que estamos terminando el semestre, y deben llevar su calendario de pruebas para que sus padres les ayuden a repasar las materias porque desde la próxima semana hay evaluaciones de las diferentes asignaturas todos los días".

Aa6: "Profesora yo la próxima semana no vengo porque vamos a viajar a Santiago".

Pa: "Jocelyn eso no puede ser, voy a tener que conversar con tus padres porque no debes faltar porque te vas a perder todas las pruebas".

Pa: "Usted Cristian ayúdeme a repartir los calendarios de pruebas y los pegan en su cuaderno, luego salgan a recreo todos aquellos que han terminado de pegar el calendario". (Dirige su mirada a los alumnos que no han terminado)

Ao9: "iTía espere me falta poco!"

(Los niños se apuran por terminar para salir a recreo).

$[\mathrm{R} 4, \mathrm{P} 2]$

La anterior actividad evaluativa refleja un débil liderazgo pedagógico, se asume la evaluación sumativa como un producto final de un proceso de enseñanza. Desdeesta perspectiva tradicional, la responsabilidad de evaluar al estudiantado recae en el personal docente, quien no propicia la evaluación de proceso en el aula, se restringe la retroalimentación oportuna y en definitiva no asume la evaluación como aprendizaje.

\section{Relación de creencias evaluativas contradictorias con prácticas de aula}

Finalmente, se presentan, en la tabla 4, algunos ejemplos de creencias contradictorias sobre evaluación del aprendizaje que comparten el cuerpo directivo y el personal docente que se relacionan con las prácticas pedagógicas observadas en el aula.

Los ejemplos de la tabla 4 nos permiten evidenciar que cuando existen ambigüedades en las concepciones pedagógicas de líderes escolares, generalmente las prácticas de aula resultan asociadas a un enfoque tradicional de enseñanza. Se observan actividades de aula que parecen estar permeadas por el pensamiento evaluativo de directivos y docentes. Estas prácticas son recurrentes en las clases observadas y se condicen con concepciones evaluativas contradictorias desde un enfoque pedagógico. 
Tabla 4

Relación de creencias evaluativas contradictorias con actividades observadas en el aula

\section{Creencias de evaluación contradictorias \\ La prueba escrita puede ser utilizada para evaluar solo ciertas habilidades. (enfoque pedagógico contemporáneo)}

La prueba escrita puede ser utilizada para evaluar cualquier tipo de habilidad. (enfoque pedagógico tradicional)

\section{Actividades evaluativas observadas en el aula}

Pa: "Niños hoy vamos a realizar la prueba escrita sobre el lenguaje figurado del poema de Neruda"

Ao3: "iUff!! Profesora! Yo no repasé porque tuve que salir con mi abuelita a un funeral.

Aa7: “¿Qué poema tía?, ¿ese que leímos del libro de lenguaje el viernes pasado?

Pa: "Recuerden que acordamos que hoy miércoles tendrían prueba de una selección de las preguntas poéticas del "Libro de las preguntas" de Pablo Neruda.

Ao1: ¿Profesora podemos leer nuevamente las preguntas y conversar para entender mejor?

Pa: "Chicos, deben entender que yo debo poner nota y para eso necesito hacer la prueba escrita". "Paulina reparta usted las pruebas y calladitos contesten para que terminen a tiempo".

(R1,P3)

El estudiantado puede participar en la construcción de los criterios de evaluación. (enfoque pedagógico contemporáneo)

El estudiantado no debe participar en la construcción de los criterios de evaluación. (enfoque pedagógico tradicional)
Po: "iBuenas días niños! Hoy vamos a leer en conjunto el cuento "La hormiguita cantora y el duende melodía" de Alicia Morel del texto de estudio".

Aa8: "iTío yo quiero leer!"

Po: "Vamos a leer todos juntos desde el telón" (leen en coro con el profesor desde internet el cuento proyectado en la pizarra)

Ao5: "Profesor Carlos ya leí este cuento en mi casa"

Po: "iQué bueno Joaquín! porque luego tendrán que contestar varias preguntas para saber si comprenden la lectura".

Aa4: ¿Tío nosotros podemos hacer preguntas?

Ao2: "Profesor, Beatriz cree que ella puede preguntar"

Po: "Tal vez cuando seas profesora Beatriz, podrás hacer las preguntas de la clase".

(R4, P4)

Nota: Elaboración propia. 
doi: http://dx.doi.org/10.15359/ree.20-1.18

URL: http://www.una.ac.cr/educare

CORREO: educare@una.cr

\section{Conclusiones}

En este artículo se puede concluir que existe una contradicción de enfoques pedagógicos sobre las creencias de enseñanza, aprendizaje y evaluación que posee tanto personal directivo como docentes que participan de este estudio. En este caso, predomina una perspectiva tradicional en sus prácticas. En otras palabras, coexisten distintos enfoques pedagógicos en el pensamiento del profesorado que se reflejan en las decisiones y acciones de enseñanza.

La transformación de las representaciones de cuerpo directivo y profesorado de un centro educativo que comparten las mismas creencias, pero contradictorias desde un enfoque pedagógico, requieren de un líder que propicie condiciones favorables para la reflexión constante sobre la práctica en función de factores conceptuales, socioculturales, políticos y económicos que le ayuden a tomar conciencia de cómo sus marcos de referencia afectan el desempeño de prácticas directivas y docentes en el aula.

Las prácticas pedagógicas del grupo de docentes en estudio responden a un enfoque de enseñanza tradicional, centradas en el currículo prescrito, aprendizaje memorístico y actividades evaluativas sumativas. Esta situación en particular, evidencia escasas competencias de liderazgo pedagógico de personal directivo y docente, asumen un pensamiento similar y confuso sobre el saber pedagógico. Por esta razón, es relevante reconceptualizar estas representaciones utilizando como estrategia la constante reflexión pedagógica entre el equipo de gestión y el profesorado.

Sin duda, se deben formar líderes escolares efectivos que brinden espacios y condiciones para transformar los centros educativos en comunidades de aprendizaje entre profesionales que permitan superar las barreras de las creencias y transformar la cultura escolar en los centros educativos. De aquí, la necesidad de aumentar la muestra y replicar este modelo investigativo a otros ámbitos tanto locales como internacionales para evidenciar, si este resultado en particular puede ser generalizado en América Latina.

\section{Referencias}

Aaronson, D., Barrow, L., y Sander, W. (2007). Teachers and student achievement in the Chicago public high schools [Los maestros y el rendimiento estudiantil en Alto de las Escuelas Públicas de Chicago]. Journal of Labor Economics, 25(1), 95-135. doi: http://dx.doi. org/10.1086/508733

Amaya, R.-A. (2007). La investigación en la práctica educativa: Guía metodológica de investigación para el diagnóstico y evaluación en los centro docentes. Madrid: Secretaría General Técnica.

Anguera, M. T., Blanco-Villaseñor, A. y Losada, J. L. (2001). Diseños observacionales, cuestión clave en el proceso de la metodología observacional. Metodología de las ciencias del Comportamiento, 3(2), 135-160. 
doi: http://dx.doi.org/10.15359/ree.20-1.18

URL: http://www.una.ac.cr/educare

CORREO: educare@una.cr

Antúnez, Á. y Aranguren, C. (Abril-junio, 2004). Problemática teórica-filosófica de la evaluación en la educación básica durante las dos últimas décadas del siglo XX. Educere, 8(25) 149153. Recuperado de http://www.redalyc.org/articulo.oa?id=35602502

Barber, M. y Mourshed, M. (Julio, 2008). Cómo hicieron los sistemas educativos con mejor desempeño del mundo para alcanzar sus objetivos. PREAL, 41, 1-48. Recuperado de http:// www.oei.es/pdfs/documento preal41.pdf

Blázquez, F.yTagle, T.(2010). Formación docente: Un estudio de creencias de alumnos y profesores sobre el proceso de enseñanza y aprendizaje del inglés. Revista lberoamericana de Educación, 54(4), 1-12. Recuperado de http://www.rieoei.org/deloslectores/3551Blazquez.pdf

Bolívar-Botía, A. (Julio-diciembre, 2010a). ¿Cómo el liderazgo pedagógico y distribuido mejora los logros académicos? Revisión de la investigación y propuesta. Magis, Revista Internacional de Investigación en Educación, 3(5), 79-106. Recuperado de http://www. redalyc.org/articulo.oa?id $=281023476005$

Bolívar-Botía, A. (Julio-diciembre, 2010b). El liderazgo educativo y su papel en la mejora: Una revisión actual de sus posibilidades y limitaciones. Psicoperpectivas, 9(2), 9-33. doi: 10.5027/ psicoperspectivas-Vol9-Issue2-fulltext-112

Clark, I. (2008). Assessment in for learning: Formative assessment and positive learning interactions [La evaluación es para el aprendizaje: Evaluación formativa e interacciones de aprendizajes positivos]. Florida Journal of Educational Administration \& Policy, 2(1), 1-16. Recuperado de http://citeseerx.ist.psu.edu/viewdoc/download?doi=10.1.1.464.3748\&re $\mathrm{p}=$ rep1\&type $=p d f$

Contreras, S. A. (2010). Las creencias y actuaciones curriculares de los profesores de ciencias de secundaria de Chile (Tesis doctoral). Universidad Complutense de Madrid, Madrid, España). Recuperada de http://eprints.ucm.es/11624/1/T32362.pdf

Costamagna, P. (2015). Política y formación en el desarrollo territorial. Aportes al enfoque pedagógico y a la investigación acción con casos de estudio en Argentina, Perú y País Vasco. Bilbao: Publicaciones Universidad de Deusto. Recuperado de http://www.orkestra. deusto.es/images/investigacion/publicaciones/informes/politica-formacion-desarrolloterritorial.pdf

Day, C. y Gu, Q. (2012). Profesores: Vidas nuevas, verdades antiguas. Una influencia decisiva en la vida de los alumnos. Madrid: Narcea.

Díaz, C., Martínez, P., Roa, I. y Sanhueza, M. G. (2010). Los docentes de la sociedad actual: Sus creencias y cogniciones pedagógicas respecto al proceso didáctico. Polis, Revista de la Universidad Bolivariana, 9(25), 421-436. Recuperado de http://www.scielo.cl/pdf/polis/v9n25/art25.pdf 
doi: http://dx.doi.org/10.15359/ree.20-1.18

URL: http://www.una.ac.cr/educare

CORREO: educare@una.cr

Erazo-Jiménez, M.S. (2009). Práctica reflexiva como estrategia de desarrollo profesional: Presencia y estructura en reuniones docente. Educación y educadores, 12(2), 47-74. Recuperado de http://www.redalyc.org/pdf/834/83412219004.pdf

García, M. R. (2010). Efecto de la formación inicial docente sobre la transformación de las creencias epistemológicas y de las creencias acerca de la buena enseñanza, de los estudiantes de Pedagogía de la Pontificia Universidad Católica de Chile (Tesis de maestría). Pontificia Universidad Católica de Chile. Recuperado de http://dspace2.conicyt.cl/bitstream/ handle/10533/76157/GARCIA MARIA 1069M.pdf?sequence=1

Gil, F., Rico, L., y Fernández., A. (2002). Concepciones y creencias del profesorado de secundaria sobre evaluación en Matemática. Revista de investigación Educativa, 20(1), 47-75. Recuperado de http://revistas.um.es/rie/article/view/97441

Gobierno de Chile. Agencia de la calidad de la educación. (2015). Informe Nacional de Resultados 2013. Ampliando la mirada de calidad educativa. Santiago, Chile: Autor. Recuperado de http://archivos.agenciaeducacion.cl/documentos-web/Informe Nacional Resultados Simce2013.pdf

Gómez, R. y Seda, I. (2008). Creencias de las educadoras acerca de la evaluación de sus alumnos preescolares: Un estudio de caso. Perfiles Educativos, 30(119), 33-54. Recuperado de http// www.redalys.org/articulo.oa?=13211156003

Gómez, V. (2010). Explorando el cambio epistemológico y conceptual en la formación inicial de profesores en distintos contextos universitarios (Informe final Proyecto FONIDE). Chile: MINEDUC.

Gómez, V., Muñoz, C., Silva, I., González, M. P., Guerra, P. y Valenzuela, J. (2014). Creencias y oportunidades de aprendizaje en la práctica educativa en contextos de pobreza. Perfiles educativos, 36(144), 173-188. doi: http://dx.doi.org/10.1016/S0185-2698(14)70630-0

Jiménez, A. B. y García, L. F. (Enero-abril, 2006). Pensar el pensamiento del profesorado. Revista Española de Pedagogía, 64(233), 105-122.

Leithwood, K. (2009). ¿Cómo liderar nuestras escuelas? Aportes desde la investigación. Santiago, Chile: Fundación Chile. Recuperado de http://www.fch.cl/wp-content/uploads/2012/08/ Libro Liethwood.pdf

Leithwood, K. y Day, C. (2007). What we learned: A broad view [Lo que aprendimos: Una visión amplia]. En C. Day y K. Leithwood (Eds.), Successful Principal Leadershirp in Times of Change: An Internacional Perspective [Liderazgo principal éxito en tiempos de cambio] (pp. 189203). Toronto: Springer. 
doi: http://dx.doi.org/10.15359/ree.20-1.18

URL: http://www.una.ac.cr/educare

CORREO: educare@una.cr

Leithwood, K., Harris, A. y Hopkins, D. (2008). Seven strong claims about successful school leadership [Siete fuertes demandas sobre el liderazgo escolar exitoso]. School Leadership and Management, 28(1), 27-42. doi: 10.1080/13632430701800060

Llinares, S. (1991). La formación de profesores de matemáticas. Sevilla: GID.

Martínez, R. D., Montero, Y. H. y Pedrosa, M. (2009). Una aproximación a las creencias epistemológicas y pedagógicas de los profesores y a su valoración de internet. Revista Iberoamericana de Educación, 51(1), 1-15. Recuperado de http://www.rieoei.org/ deloslectores/3138Martlnez.pdf

Mellado, M. E. y Chaucono, J. C. (Setiembre-diciembre, 2015). Creencias pedagógicas del profesorado de una escuela rural en el contexto mapuche. Actualidades Investigativas en Educación, 15(3), 1-19. doi: http://dx.doi.org/10.15517/aie.v15i3.20924

Moreno, J. (2000). El profesor universitario de Matemáticas: Estudio de las concepciones y creencias acerca de la enseñanza de las ecuaciones diferenciales. Estudio de casos (Tesis de doctorado). Universidad Autónoma de Barcelona, España.

Mulford, B. (2006). Liderazgo para mejorar la calidad de la educación secundaria. Algunos desarrollos internacionales. Profesorado. Revista de Currículum y Formación del Profesorado, 10(1), 1-22. Recuperado de https://www.ugr.es/ recfpro/rev101ART2.pdf

Murillo, F. J. (2006). Una dirección escolar para el cambio: Del liderazgo transformacional al liderazgo distribuido. Revista Electrónica Iberoamericana sobre Calidad, Eficacia y Cambio en Educación, 4 (4e), 11-24. Recuperado de http://www.rinace.net/arts/vol4num4e/art2.pdf

Prieto, M. (Junio, 2008). Creencias de los profesores sobre evaluación y efectos incidentales. Caracas, Venezuela. Revista de Pedagogía, 29(84), 123-144. Recuperado de http://www. redalyc.org/articulo.oa?id $=65908405$

Rivkin, S. G., Hanushek, E. A. y Kain, J. F. (Marzo, 2005). Teachers, schools, and academic achievement [Maestros, escuelas y el rendimiento académico]. Econométrica, 73(2), 417458. Recuperado de http://www.econ.ucsb.edu/ jon/Econ230C/HanushekRivkin.pdf

Rojas, M. T. (2014). Las creencias docentes: Delimitación del concepto y propuesta para la investigación. Revista Electrónica Diálogos Educativos, 14(27), 89-112. Recuperado de http://www.dialogoseducativos.cl/revistas/n27/rojas

Sun, Y., Correa, M., Zapata, A. y Carrasco, D. (2011). Resultados: Qué dice la evaluación docente acerca de la enseñanza en Chile. En J. Manzi, R. González y Y. Sun (Eds.), La evaluación docente en Chile (91-135). Santiago de Chile: Mide UC. 
doi: http://dx.doi.org/10.15359/ree.20-1.18

URL: http://www.una.ac.cr/educare

CORREO: educare@una.cr

Vera, D. y Meneses, P. (Julio-diciembre, 2012). Construcción de una escala para medir creencias acerca del proceso educativo en profesores rurales. Liberabit, 18(2), 183-193. Recuperado de http://www.scielo.org.pe/pdf/liber/v18n2/a09v18n2.pdf

\section{(0) Cómo citar este artículo en APA:}

Mellado-Hernández, M.E.y Chaucono-Catrinao, J.C. (Enero-abril, 2016). Liderazgo pedagógico para reestructurar creencias docentes y mejorar prácticas de aula en contexto mapuche. Revista Electrónica Educare, 20(1), 1-18. doi: http://dx.doi.org/10.15359/ree.20-1.18

Nota: Para citar este artículo en otros sistemas puede consultar el hipervínculo "Como citar el artículo" en la barra derecha de nuestro sitio web: http://www.revistas.una.ac.cr/index.php/EDUCARE/index 